\title{
Real-time single analog output for quadrature phase interferometry
}

\author{
C Barraud, L Garcia, B Cross, E Charlaix \\ Univ. Grenoble Alpes, LIPHY, F-38000 Grenoble, France CNRS, LIPHY, \\ F-38000 Grenoble, France \\ E-mail: elisabeth.charlaix@univ-grenoble-alpes.fr
}

\begin{abstract}
We present a dynamic displacement sensor based on a quadrature phase interferometer, providing a real-time analog output of the differential displacement between two mobile surfaces. The sensor offers a sub-picometer resolution with a sensitivity essentially uniform over a distance range extending to several micrometers, and can be used to measure the amplitude and phase of very small oscillations in nano-mechanical testing. We demonstrate its use in nano-rheology, by studying the flow in nanometric liquid films at very small strain rates.
\end{abstract}

PACS numbers: 07.60.Ly; 06.30.Bp

Keywords: interferometry, nanometrology, nanomechanics

Submitted to: Meas. Sci. Technol. 


\section{Introduction}

Dimensional measurements play an important role in many industrial and instrumentation applications[1]. In nano-mechanical and rheological measurements, it is important to apply and to measure oscillatory motions of very low amplitude in order to probe the visco-elastic modulii of nano-scale objects at a vanishing small strain, without impacting their molecular or interfacial arrangements. This is the case in the determination of the rheological properties of confined liquids $[2,3,4,5]$ or in the measurement of the mechanical properties of polymer coatings $[6,7]$ or in the characterization of the slippage of flows on nanobubbles, surfactants, lipid membranes $[8,9,10,11]$ as examples.

Homodyne interferometry with quadrature phase detection has become a leading technique in metrological distance measurements, enabling resolutions of some picometers over millimetric range [12]. It is used on metrological AFM [13, 14] and has been implemented for nano-mechanical measurements $[15,6]$. However quadrature phase detection involves two signals in which the dimensional measurement is encoded, and its extraction usually involves digital processing. Therefore it is not readily usable in lock-in amplification techniques to probe the linear mechanical response of nano-systems. For these real-time applications it is of interest to have a single, linear analog output of the dimensional measurement such as offered for instance by capacitive sensors $[16,17]$.

Recent advances have been made on the real-time extraction of the optical phase shift in quadrature phase interferometry, using fast analog-to-digital converters and optimized calculation schemes for nonlinearity corrections $[18,19,20,21]$. Here we propose a simple, low-cost analog demodulation device which provides a real-time linear output of the dimensional measurement over four orders of magnitude from the picometer to 10 nanometers. The sensitivity of this analog output is essentially constant over an arbitrary wide range, with less than $2 \%$ cyclic variation with respect to the working point. The bandpass of the device has been designed to produce negligible phase distorsion over a frequency range 20-400 $\mathrm{Hz}$ matching the mechanical capabilities of our dynamic Surface Force Apparatus, but it can be easily tuned to meet other requirements. We illustrate the use of this sensor to measure nano-hydrodynamic forces in liquid films from the nanometer to several micrometers thickness, driven in the linear regime with oscillations amplitude no larger than the hundredth of their nominal thickness.

\section{Principle of the real-time analog sensor}

A quadrature homodyne interferometer (see our example fig. 2) provides two interference signals $C_{x}$ and $C_{y}$ shifted in the ideal case by $90^{\circ}$, to allow a detection of motion over a wide distance range without periodic fading of the sensitivity:

$$
C_{x}=A_{x} \cos \varphi \quad C_{y}=A_{y} \sin \varphi \quad \varphi=\frac{4 \pi d}{\lambda}
$$

In eq. (1) $\varphi$ is the optical phase shift of the interfering beams, $\lambda$ the wavelength of light, and $d$ the dimension to be measured. Both signals are non-linear functions of the optical phase $\varphi$. We are here interested in an analog signal giving access in real time to small variations $\delta \varphi(t) \ll 2 \pi$ around a current working point $\bar{\varphi}$, for feeding lock-in amplifiers in nano-mechanical testing, feed-back loops, or vibration control. The working point should possibly be changed over a wide scale for enabling large operating distances (from the nanometer to tens of micrometers in our application) but on a time scale slower than the variations of $\delta \varphi(t)$ of interest ( $20 \mathrm{~Hz}$ to $400 \mathrm{~Hz}$ in our application).

A method to demodulate analogically homodyne interferometry signals for displacement or velocity measurements is to perform the operation $C_{x} \times$ $d C_{y} / d t-C_{y} \times d C_{x} / d t \sim d \varphi / d t$ and to integrate the result [22]. We propose a real time displacement sensor based on the electronic operation:

$\widehat{C_{x}} C_{y}(t)-\widehat{C_{y}} C_{x}(t)$

where $\widehat{C_{x}}=C_{x}(\bar{\varphi})$ and $\widehat{C_{y}}=C_{y}(\bar{\varphi})$ are the low-pass filtered contrast signals. For an ideal interferometer, we expect the analog signal produced by the eq. 2 to reproduce the temporal variations $\delta \varphi(t)$ with a uniform sensitivity:

$$
\begin{aligned}
\frac{\widehat{C_{x}} C_{y}(t)-\widehat{C_{y}} C_{x}(t)}{\delta \varphi(t)} & =\widehat{C_{x}} C_{y}^{\prime}(\bar{\varphi})-\widehat{C_{y}} C_{x}^{\prime}(\bar{\varphi}) \\
& =A_{x} A_{y}
\end{aligned}
$$

where $C_{x}^{\prime}(\bar{\varphi})$ and $C_{y}^{\prime}(\bar{\varphi})$ are the derivative of the contrasts with respect to the optical phase taken at the working point $\bar{\varphi}$.

We perform the analog of operation (2) using the homemade circuit shown in figure (1). The raw contrasts $C_{x}^{\text {raw }}$ and $C_{y}^{\text {raw }}$ delivered by a quadrature phase interferometer can be compensated by two offsets $O_{x}^{\text {cor }}$ and $O_{y}^{\text {cor }}$ to deliver two symmetric contrasts $C_{x}(t)$ and $C_{y}(t)$. A low-pass filter is used to produced $\widehat{C_{x}}$ and $\widehat{C_{y}}$. The implemented filter is a Sallen-key filter realizing a second-order filter. The cutoff frequency is set at $1 \mathrm{~Hz}$, a time scale convenient in our application to follow slow changes of the working point. The circuit delivers a single analog voltage

$U(t)=\frac{\widehat{C_{x}} C_{y}(t)-\widehat{C_{y}} C_{x}(t)}{K}$

with $\mathrm{K}=10 \mathrm{~V}$ is the gain of the AD734 multiplier. 


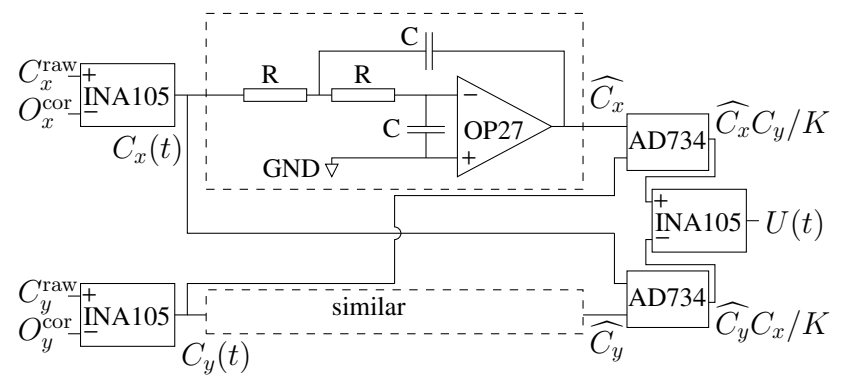

Figure 1. Schematic of the analog circuitry. The integrated circuits references to make analog electronic operations are given on the schematic. The resistance $\mathrm{R}$ is equal to $318 \mathrm{k} \Omega$ and the capacitance $\mathrm{C}$ is equal to $470 \mathrm{nF}$.

\section{Optical implementation}

We study this dynamic displacement sensor on a Nomarsky interferometer mounted on our dynamic Surface Force Apparatus as sketched in figure 2 (see also $[23,24,25])$. Two surfaces, a plane and a sphere are separated by a distance $D$. The plane is mounted on a a piezo-electric stage (Physik Instrument Lisa P753) which enables us to change slowly the nominal distance $D$ between the surfaces and to drive an oscillatory displacement of very low amplitude $h_{o}$ at a frequency $f=\omega / 2 \pi$ between $20 \mathrm{~Hz}$ and $400 \mathrm{~Hz}$. The sphere is mounted on a cantilever, whose deflection gives access to the force in our dSFA.

To measure the relative displacement between the surfaces, the optical implementation is made as follow: a calcite prism is used to separate the two polarizations $\mathrm{s}$ and $\mathrm{p}$ of a He-Ne laser beam initially polarized at $45^{\circ}$ with respect to the calcite axis. The output beams impinge on two mirrors, separated by a distance $d$. One is rigidly fixed on the plane and the other is rigidly fixed on the sphere. In the following we note

$d(t)=D+d_{o}+h_{o} \cos (\omega t)=\frac{\lambda(\bar{\varphi}+\delta \varphi(t))}{4 \pi}$

$\bar{\varphi}=\frac{4 \pi D}{\lambda}+\varphi_{o} \quad \delta \varphi(t)=\frac{4 \pi h_{o}}{\lambda} \cos (\omega t)$

where $d_{o}$ is the distance between the mirrors when the surfaces are in contact $(\mathrm{D}=0)$.

The analysis part of the interferometer is made of two arms as described in Bellon[15] and Paolino et al.[26] and represented in figure 2. Each arm includes a Wollaston prism $W_{x}$ and $W_{y}$ oriented at $45^{\circ}$ with respect to the calcite axes, and a pair of photodiodes (Osram Opto SFH $206 \mathrm{~K}$ ) mounted in photovoltaic mode. The $y$ arm also includes a quarter wave plate oriented so that it subtracts $\pi / 2$ to the optical phase in this arm. A home-made low-noise analog amplifier enables us to access to the raw contrasts signals

$C_{i}^{\mathrm{raw}}=K \frac{I_{1, i}-I_{2, i}}{I_{1, i}+I_{2, i}} \quad i=(x, y)$ where $K=10 \mathrm{~V}$ is the gain of the amplifier and $I_{1 / 2, i}$ are the photovoltaic currents delivered by the photodiodes.

The optical setup is made of a He-Ne laser (Melles Griot 05-STP-912) of wavelength $\lambda=632.8 \mathrm{~nm}$, with a frequency stability of $1 \mathrm{MHz}$ over 8 hours. The laser beam passes through a Faraday isolator (Newport ISO04-650-LP) and through a polarization maintaining optical fiber (Newport F-PM630 FC/APC, $4.5 \mu \mathrm{m}$ core diameter) whose end is connected to a collimator (Newport F-H10-NIR APC). The polarization of the initial laser beam is tuned thanks to a Glan-Taylor polarizer (Thorlabs DGL10) with very high extinction ratio $(100,000: 1)$.

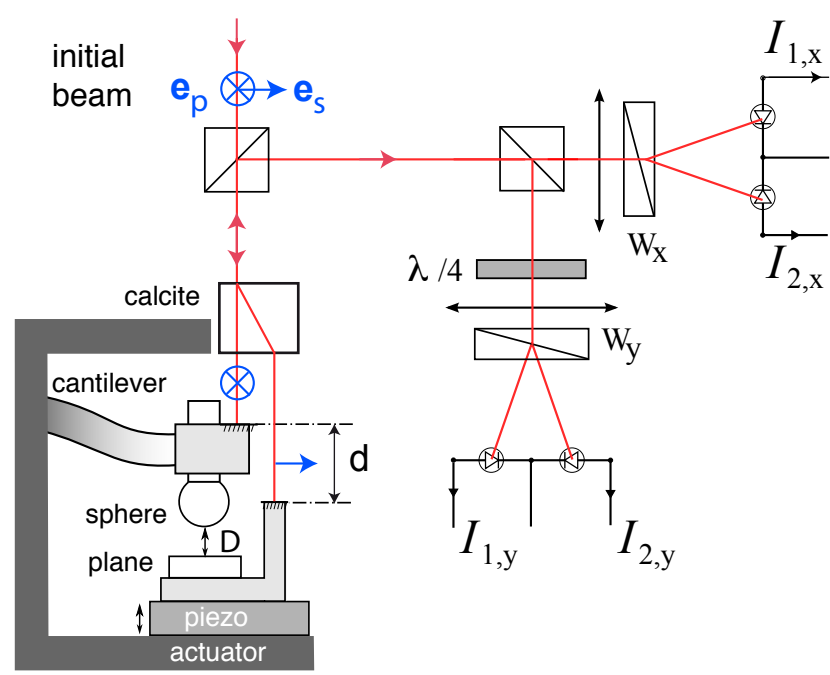

Figure 2. Schematic of the Nomarski quadrature phase interferometer implemented on the Surface Force Apparatus

In order to study our analog dynamic sensor, the contrasts $C_{x}$ and $C_{y}$ (eventually compensated by the offsets) must first be characterized as a function of the optical phase shift $\varphi$ of the interfering beams. For this we move slowly the piezoelectric stage while keeping the flexure hinge immobile. The two signals $C_{x}$ and $C_{y}$ are acquired and averaged by two digital multimeters (Agilent 34410) triggered with a $2 \mathrm{~Hz}$ TTL signal. One point of measurement corresponds to a voltage averaged over the first $300 \mathrm{~ms}$ of the trig period.

The measured contrats are plotted in the Lissajous mode to determine the so-called Heydemann corrections $[27,28]$ due to the optical and electronic imperfections:

$C_{x}=O_{x}+A_{x} \cos (\varphi)+\epsilon_{x}(\varphi)$

$C_{y}=O_{y}+A_{y} \sin (\varphi+\psi)+\epsilon_{y}(\varphi)$

where $\epsilon_{i}(\varphi)$ account for small systematic higher-order non-linearities. In particular the phase lag $\psi$ is due to the phase mixing induced by the imperfection of optical components. Here we have amplified this 


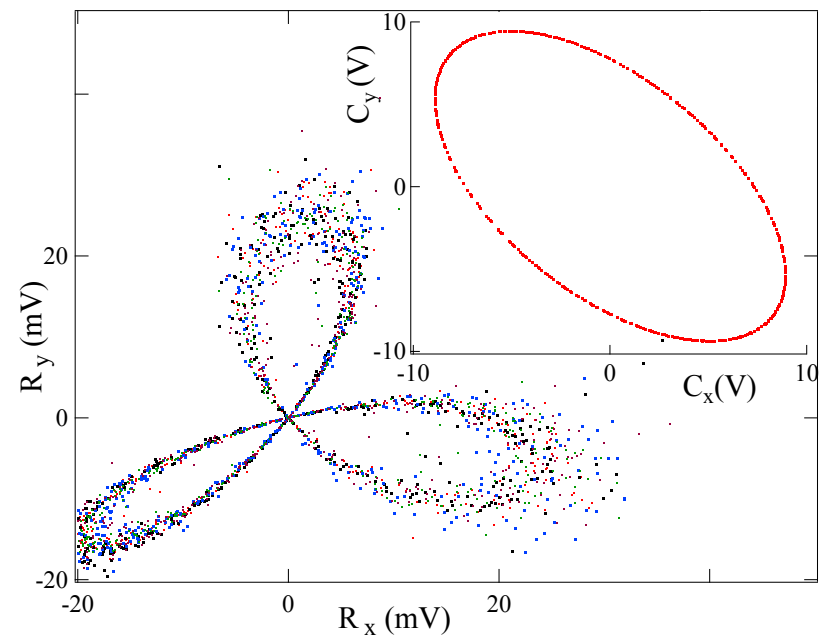

Figure 3. Static calibration of the interferometer. Inset: contrasts $C_{x}$ and $C_{y}$ measured in the $x$ and $y$-arm, in Volt, plotted in the Lissajous mode. Main plot: residuals of the best fit of $C_{x}$ and $C_{y}$ to a generic ellipse, in mVolts, plotted in Lissajous mode.

phase mixing on purpose by misaligning the Wollaston prism with respect to the calcite, in order to test the robustness of our dynamic sensor with respect to optical imperfections.

We determine the 5 coefficients of equation (7) by fitting the data to an ellipse in the Igor Pro software. The residuals of the fit are plotted in fig. 3 in the Lissajous mode. They have a relative amplitude less than $\pm 310^{-3}$ of the maximum nominal signals. The plot has the general shape of a trifolium, showing the existence of systematic higher-order non-linearities $\epsilon_{x}(\varphi)$ and $\epsilon_{y}(\varphi)$ in $2 \varphi$ and $4 \varphi$. These higher order non-linearities are likely due to electronic imperfections in the normalization of the signals by the total light intensity (eq. 6).

In the following, we determine the value of the nominal optical phase shift $\bar{\varphi}$ by inverting Eq. 7 without the higher harmonic terms:

$\bar{\varphi}=\operatorname{atan}\left(\frac{\left(C_{x}-O_{x}\right) A_{y}}{\left(C_{y}-O_{y}\right) A_{x}}-\tan \psi\right)$

with $C_{i}$ the contrasts measured by the multimeters. The systematic error $\bar{\varphi}_{\text {err }}$ made on $\bar{\varphi}$ by neglecting the higher-order harmonics in Eqs.(7) can be evaluated from a first-order development as:

$$
\bar{\varphi}_{e r r}=\left(\frac{\epsilon_{x}\left(C_{y}-O_{y}\right)-\epsilon_{y}\left(C_{x}-O_{x}\right)}{A_{x} A_{y} \cos (\psi)}\right)
$$

The amplitude of $\bar{\varphi}_{\text {err }}$ is very small and always lower than $\pm 5 \cdot 10^{-6}$ rad. It is much lower than the noise due to environmental vibrations, estimated using the calculation of Koning et al $[29,30]$ as:

$$
\varphi_{\text {noise }}=\frac{\sigma}{A} \frac{\sqrt{1+|\sin \psi|}}{\cos \psi} \sim 10^{-3}
$$

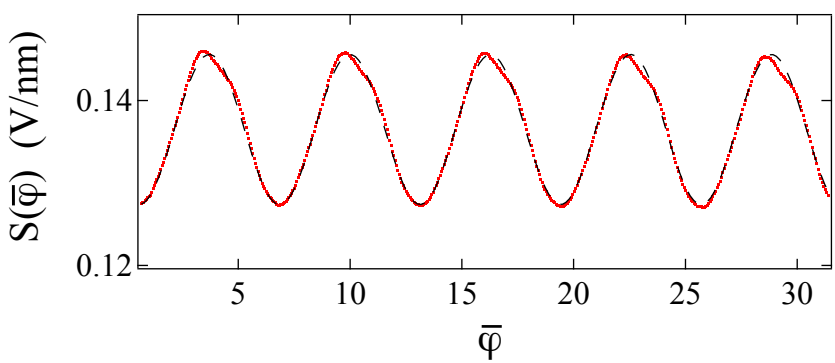

Figure 4. Sensitivity in Volt/nm of the real-time analog sensor as a function of the working point, characterized by the average value of the optical phase shift $\bar{\varphi}$. The dashed line is the best fit with a sinusoidal variation in $\bar{\varphi}$.

were $A / \sigma \simeq 2.10^{3}$ is the signal-to-noise ratio. Therefore the systematic error on $\bar{\varphi}$ can be neglected, and the determination of the working point distance is made with an uncertainty of $50 \mathrm{pm}$ in our bandwidth of $2 \mathrm{~Hz}$.

\section{Metrologic characteristics of the real-time sensor.}

\subsection{Sensitivity}

We study the real-time analog output by driving the piezoelectric stage with the harmonic source of a SR830 lock-in amplifier, the flexure hinge being kept immobile.

We first calibrate the amplitude of the harmonic displacement (see eq. 5 for notations) by moving slowly the stage until we reach a position such that $\widehat{C}_{x} \approx 0$, and by analyzing the temporal signal $C_{x}(t)$ with the lock-in amplifier. The sensitivity of the $x$-contrast $\delta C_{x} / \delta \varphi=-A_{x} \sin (\bar{\varphi})$ is thus close to its maximum, and we obtain the amplitude $\delta \varphi$ of the optical phase oscillation from the quasi-static calibration above. Then, while keeping the same driving amplitude, the nominal position $\bar{\varphi}$ is varied slowly at a maximum speed of $1 \mathrm{~nm} / \mathrm{s}$, and the amplitude of $U(t)$ analyzed with the lock-in amplifier is recorded on several periods of $\bar{\varphi}$. The experimental sensitivity $S(\bar{\varphi})$ is obtained by dividing this amplitude by $\delta \varphi$.

Figure 4 plots the sensitivity as a function of the nominal optical phase $\bar{\varphi}$. We observe that $S$ varies periodically around an average value, with peak-topeak amplitude about $5 \%$ of this average sensitivity. This variatons are due to the non-ideality of our optical implementation. More specifically, the theoretical sentivity (3) calculated using the equations (7) giving the actual contrasts of our interferometer, reproduces quite well the periodic variations observed. 


\subsection{Offsets compensation}

Using the expressions 7 and neglecting in a first approach the non-linear terms, we get the following theoretical expression for the sensor's sensitivity:

$S^{t h}=\frac{U}{\delta \varphi}=\left(A_{x} A_{y} \cos \psi\right.$

$\left.+O_{x} A_{y} \cos (\bar{\varphi}+\psi)+O_{y} A_{x} \sin \bar{\varphi}\right) / K$

with $\mathrm{K}=10 \mathrm{~V}$ the gain of the multiplier. It appears clearly that the offsets $O_{x}$ and $O_{y}$ of the contrast signals are leading terms in the sinusoidal variation of $S(\bar{\varphi})$ with $\bar{\varphi}$ and should be kept as low as possible to obtain a sensitivity not depending on the working point.

For this purpose we have equipped our analog circuit with two entries allowing us to substract a constant voltage $O_{x}^{\text {cor }}$ and $O_{y}^{\text {cor }}$ to the raw contrast signals $C_{x}^{\text {raw }}$ and $C_{y}^{\text {raw }}$. Figure 5 shows the measured sensitivity with the offsets compensation, plotted as a function of the working point in the same conditions of oscillatory driving as above. After adding $O_{x}^{\text {cor }}$ and $O_{y}^{\text {cor }}$ to the raw contrasts, the residual offsets of the resulting contrasts $C_{x}$ and $C_{y}$ in this experiment are $O_{x}=0.5 \mathrm{mV}$ and $O_{y}=2.5 \mathrm{mV}$. The peak-to-peak variations of $S(\bar{\varphi})$ around its average value are now less than $1.5 \%$ of its average value. These variations are higher harmonics in $n \bar{\varphi}, n \geq 2$, and are mainly due to the higher-order non-linearities $\epsilon_{x}(\varphi)$ and $\epsilon_{y}(\varphi)$ studied above (figure 3). More specifically, taking quantitatively into account these higher-order terms in eq. 7 , we can estimate a theoretical sensitiviy which reproduces the main features of the measured sensitivity. Note that although the higher-order nonlinearities in our interferometer represent no more than $\pm 2.10^{-3}$ of its full scale contrasts, they result in cyclic variations of the sensitivity of the real-time analog output of about $1.5 \%$ of its average value, even with the offsets compensation. The best results are obtained for values of the residual offsets $O_{x}$ and $O_{y}$ after compensation, which are not exactly zero but up to $\pm 2.10^{-4}$ of the full scale contrats (that is, millivolts in our case).

In our SFA operation, we calibrate the residual variations of $S(\bar{\varphi})$ as a function of $\bar{\varphi}$ as shown in figure 5 , in order to achieve a relative precision better than $1 \%$ in the measurement of the gap oscillation of the two surfaces.

\subsection{Noise and resolution}

Figure 6 shows the magnitude of the intrinsic noise on a wide frequency range. Apart from the peaks specific to the mechanical environment of the SFA, the average level of noise is lower than $10^{-12} \mathrm{~m} / \sqrt{\mathrm{Hz}}$ (RMS value), reaching less than $10^{-13} \mathrm{~m} / \sqrt{\mathrm{Hz}}$ (RMS value) for frequencies higher than $3 \mathrm{kHz}$. The main

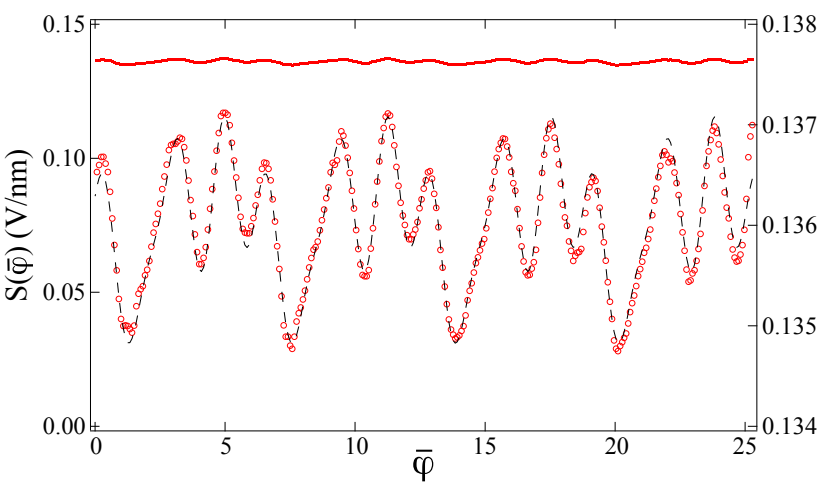

Figure 5. Sensitivity in Volt/nm of the analog sensor with the offsets compensation, as a function of the optical phase shift $\bar{\varphi}$ of the working point. Left axis: full scale. Right axis: variation of $S(\bar{\varphi})$ around its average value of $136 \mathrm{mV} / \mathrm{nm}$. The symbols (o) is the data. The dashed line is the best fit with a periodic function of $\bar{\varphi}$.

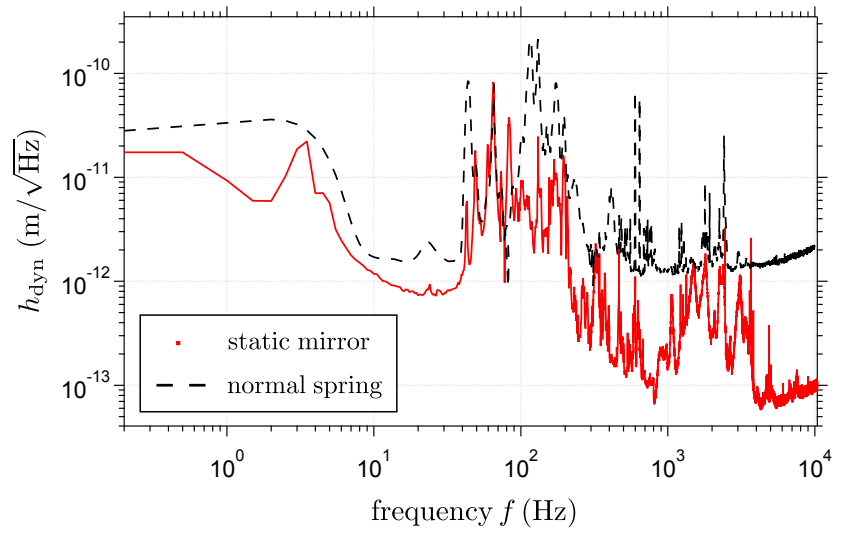

Figure 6. Linear spectrum magnitude of the dynamic sensor (in $\mathrm{m} / \sqrt{\mathrm{Hz}}$, RMS value) when i) the two moving surfaces are rigidily fixed to the frame (continuous line) and ii) the cantilever holding the sphere is free to move (dashed line).

contribution to this background noise is the shot noise due to photodiodes, whereas the higher value at low frequency is attributed to the electronics implemented for the measurement of $h$.

Figure 6 also presents the spectrum magnitude in the regular SFA configuration (free normal spring) obtained in the same conditions in terms of photodiodes lighting. The environmental vibrations probed by the normal spring (whose resonance peak is at $f=145 \mathrm{~Hz}$ ) bring about an average noise that exceeds the intrinsic noise of the sensor, but remains lower than $10^{-11} \mathrm{~m} / \sqrt{\mathrm{Hz}}$ (RMS value) and can reach less than $10^{-12} \mathrm{~m} / \sqrt{\mathrm{Hz}}$ (RMS value) for several frequencies. With the $24 \mathrm{~dB}$ amplification of the lock-in amplifier and an integration time of $0.3 \mathrm{~s}$, the dynamical amplitude $h_{0}$ can thus be measured with a very high average precision of $5 \mathrm{pm}$ in the equivalent noise bandwidth during the SFA experiments. This precision 


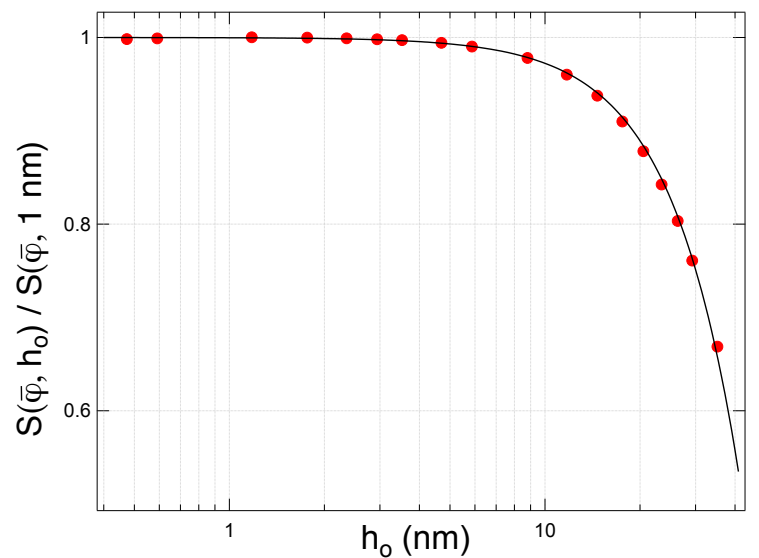

Figure 7. Non-linear calibration of the dynamic sensor as a function of the amplitude $h_{0}$ of the oscillatory displacement. Markers are experimental data points which represent the measured optical sensitivity over the sensitivity measured for a displacement of $1 \mathrm{~nm}$, the continuous line is the best fit with the function $1+\alpha h_{0}^{2}$.

can even reach $0.2 \mathrm{pm}$ for optimal frequencies (e.g. 75 and $220 \mathrm{~Hz}$ ), which are thus our typical working frequencies when using the SFA.

\subsection{Linearity}

As the dynamic sensor is based on interferometric contrasts its range of linearity is limited. However, it is still possible to work with larger oscillation amplitudes by calibrating the nonlinear behavior of the sensor. For this, we release the sphere and we force the oscillation of the cantilever holding it, thanks to a coil-magnet system. As the amplitude of the sphere oscillation is strictly proportional to the amplitude of the driving current, we can have access to the actual value of the amplitude $h_{0}$ even when the dynamic sensor gets off its linear range. As shown in Figure 7, the measured sensitivity decrease for an increase of the displacement $h_{0}$. This sensitivity normalized by the sensitivity at $1 \mathrm{~nm}$ of displacement can be fitted with a function $1+\alpha h_{0}^{2}$, which corresponds to the expected behavior considering the second order development in $\delta \varphi$ of the quantity $\left(\widehat{C}_{x} C_{y}(\bar{\varphi}+\delta \varphi)-\widehat{C}_{y} C_{x}(\bar{\varphi}+\delta \varphi)\right) / \delta \varphi$. Calibrating the coefficient $\alpha$ enables us to work with amplitudes as high as $h_{0} \approx 40 \mathrm{~nm}$ with more than $50 \%$ of the sensitivity. From this calibration we can define the linear regime where the sensitivity is independent of the displacement $h_{o}$. For a deviation less than $1 \%$ from the linear behavior, the displacement $h_{o}$ has to be lower than $6 \mathrm{~nm}$.
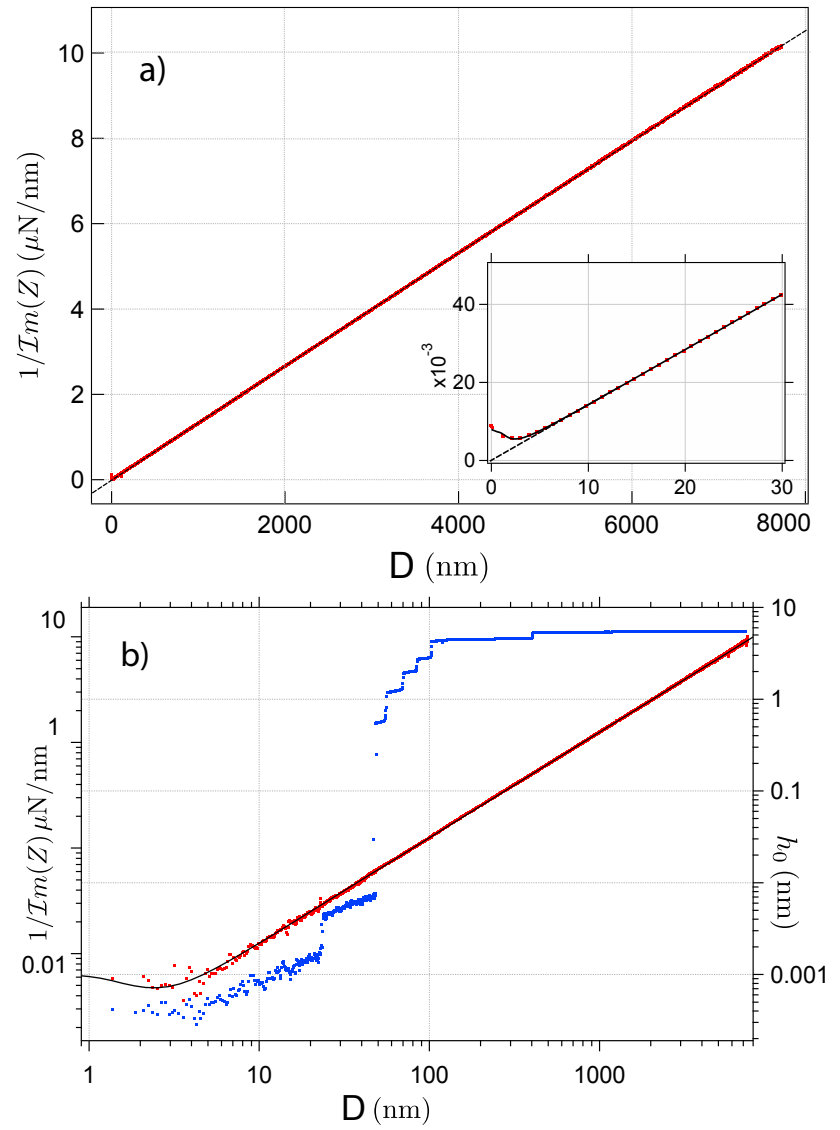

Figure 8. Plot of the inverse of $\operatorname{Im}(Z)$ (red points) as a function of the nominal sphere-plane distance $D$ in n-hexadecane. a) linear scale, b) log-scale. The driving frequency is $220 \mathrm{~Hz}$ and the constant drift velocity is $1 \mathrm{~nm} / \mathrm{s}$. The amplitude of the harmonic oscillation $h_{0}$ is plotted on the right axis of b) (blue dots). The origin is chosen so that $1 / \operatorname{Im}\left(Z^{\prime \prime}\right)$ extrapolate to 0 . For $D>7 \mathrm{~nm}$, the linear behavior is in good agreement with Eq. 10. For $D<7 \mathrm{~nm}$, deviations occur. They are well described by the elastohydrodynamic deformation of the glass surfaces [31] (black solid line in b)).

\section{Application to nanohydrodynamics}

We illustrate the use of this real time sensor to study the nano-rheological behavior of a thin liquid film in the SFA setup of fig. 2. The piezoelectric stage is used to change slowly the sphere-plane distance $D$ and to drive a small amplitude oscillation $h_{0} \cos \omega t$ of this distance (see eq. 5 for notations). Those quantities are measured by the quadrature phase sensor. The complex amplitude $F_{a c}$ of the oscillatory force acting on the sphere is deduced from the spring deflection $x_{a c}$ (after calibrating the spring dynamic response with the coil magnet system) with $x_{a c}$ measured by a Normarski interferometer [25]. The complex mechanical impedance $Z=F_{a c} / h_{o}$ is thus experimentally determined. In the case of no-slip boundary condition and perfectly rigid surfaces, the 
expected impedance is purely imaginary and writes:

$Z=\frac{F_{a c}}{h_{0}}=j \frac{6 \pi \eta \omega R^{2}}{D_{H}}$

with $\eta$ the fluid bulk viscosity, $R$ the sphere radius (typ. $R \approx 3.0 \mathrm{~mm}$ ), $\omega=2 \pi f$ the angular frequency, and $D_{H}$ the distance between the no-slip planes.

Figure 8 plots the inverse of the imaginary part of $Z$ as a function of $D$ at a driving frequency of $220 \mathrm{~Hz}$. The experiment is performed on a liquid film of hexadecane confined between ultra-smooth borosilicate surfaces. The plane is driven toward the sphere at a constant velocity of $1 \mathrm{~nm} / \mathrm{s}$ over the total piezo actuator expansion range of about $8 \mu \mathrm{m}$. For large separation, the data corresponds perfectly to the expected linear behavior, and allows one to measure the liquid bulk viscosity as well as the hydrodynamic origin.

As the liquid film thickness $D$ becomes smaller, the amplitude $h_{0}$ of the oscillating displacement has to be gradually reduced in order to probe the liquid in the linear response limit. The figure $8 \mathrm{~b}$ shows the value of $h_{0}$ in this experiment. The decrease of 2 orders of magnitude of $h_{0}$ at $D=50 \mathrm{~nm}$ illustrates the linearity of the response. For film thicknesses $D<10$ $\mathrm{nm}$ we reach oscillation amplitudes of a fraction of a picometer, and still measure the mechanical response accurately. For film thickness $D<7 \mathrm{~nm}$ the inverse of the damping does not anymore follow the linear variation (10), due to the elastic deformation of the glass surfaces under the lubrication pressure [32]. The measured damping is in excellent agreement with the elastohydrodynamic theory (black line) that takes into account this deformation[31], without any ajustable parameter.

\section{Conclusion}

Our dynamic sensor, based on a quadrature phase Nomarski interferometer, measures the displacements of mobile surfaces in real-time with a resolution less than one picometer, without need of digital postprocessing. It can operate with nominal distances of several micrometers. It is thus appropriate for lockin amplification methods in nanomechanical testing of objects of various sizes, and for control signal in feedback loops. The sensitivity of the sensor can be made independent of the relative position of the surfaces, by appropriate electronic compensation of offsets. The calibration is stable and holds on a oneday time scale. The sensor is linear within $1 \%$ up to an amplitude of $6 \mathrm{~nm}$. With a non-linear calibration, it can be used up to amplitude of vibrations of $40 \mathrm{~nm}$, with more than $50 \%$ of the sensitivity.

\section{Acknowledgments}

We thank Ludovic Bellon for helpfull discussions, and Jean-Luc Mocellin from Institut Neel in Grenoble for advices in the electronic design. This work has been partially supported by contract ANR-14-CE05-0017-02 of the Agence Nationale de la Recherche in France, and by the CNRS-DEFI Instrumentation.

[1] M. Pisani. A homodyne michelson interferometer with subpicometer resolution. Meas. Sci. Technol., 20:084008, 2009.

[2] S.H. Khan, E.L. Kramkowski, P.J. Ochs, D.M. Wilson, and P.M. Hoffmann. Viscosity of a nanoconfined liquid during compression. Appl. Phys. Lett., 104:023110, 2014.

[3] A. Labuda, K. Kobayashi, K. Suzuki, H. Yamada, and P. Grutter. Monotonic damping in nanoscopic hydration experiments. Phys. Rev. Lett., 110(066102), 2013.

[4] F. Lapique, J.-P. Montfort, and C. Derail. Nanorheology of adsorbed polymer chains immersed in pure solvent. Eur. Phys. J. E, 38:58, 2015.

[5] A. Maali, T. Cohen-Bouhacina, G. Couturier, and J.P. Aimé. Oscillatory dissipation of a simple confined liquid. Phys. Rev. Lett., 96(086105), 2006.

[6] P. Paolino and L. L Bellon. Frequency dependence of viscous and viscoelastic dissipation in coated microcantilevers from noise measurement. Nanotechnology, 20:405705, 2009.

[7] S. Leroy, A. Steinberger, C. Cottin-Bizonne, F. Restagno, L. Léger, and E. Charlaix. Hydrodynamic interaction between a spherical particle and an elastic surface: A gentle probe for soft thin films. Phys. Rev. Lett., 108:264501, 2012.

[8] O. Manor, I.U. Vakarelski, X. Tang, S. J. O'Shea, G. W. Stevens, F. Grieser, R. R. Dagastine, and D.Y.C. Chan. Hydrodynamic boundary conditions and dynamic forces between bubbles and surfaces. Phys. Rev. Lett., 2008.

[9] B. Cross, A. Steinberger, C. Cottin-Bizonne, J.P. Rieu, and E. Charlaix. Boundary flow of water on supported phospholipid films. Europhys. Lett., 73(3):390-395, 2006.

[10] T. Lee, E. Charrault, and C. Neto. Interfacial slip on rough, patterned and soft surfaces. Adv. in Coll. and Interf. Sci., 210:21-38, 2014.

[11] A. Steinberger, C. Cottin-Bizonne, P. Kleimann, and E. Charlaix. Nanoscale flow on a bubble mattress: Effect of surface elasticity. Phys. Rev. Lett., 100:134501, 2008.

[12] M. Pisani, A. Yacoot, P. Balling, N. Bancone, C. Birlikseven, M. Celik, J. Flugge, R. Hamid, P. Kochert, P. Kren, U. Kuetgens, A. Lassila, E. Picotto, G. B. andSahin, J. Seppa, M. Tedaldi, and C. Weichert. Comparison of the performance of the next generation of optical interferometers. Metrologia, 49:455-467, 2012.

[13] G. Dai, F. Pohlenz, H.-U. Danzebrink, K. Hasche, and G. Wilkening. Improving the performance of interferometers in metrological scanning probe microscopes. Meas. Sci. Technol., 15:444-450, 2004.

[14] G. Dai, H. Wolff, F. Pohlenz, and H.-U. Danzebrink. A metrological large range atomic force microscope with improved performance. Rev. Sci. Instrum., 80:043702, 2009.

[15] L. Bellon, L. Buisson, S. Ciliberto, and F. Vittoz. Zero applied stress rheometer. Rev. Sci. Inst., 73(9):3286, 2004.

[16] A. Tonck, J.M. Georges, and J. L. Loubet. Measurements of intermolecular forces and the rheology of dodecane between alumina surfaces. Journal of Colloid and Interface Science, 126, 1:150-163, 1988. 
[17] F. Restagno, J. Crassous, E. Charlaix, and M. Monchanin. A new capacitive sensor for displacement measurement in a surface-force apparatus. Measurement Science and Technology, 12(1):16, 2001.

[18] O. Cip and F. Petru. A scale-linearization method for precise laser interferometry. Meas. Sci. Technol., 11:133$141,2000$.

[19] T. Keem, S. Gonda, I. Misumi, Q. Huang, and T. Kurosawa. Simple, real-time method for removing the cyclic error of a homodyne interferometer with a quadrature detector system. Applied Optics, 44(17):3492, 2005.

[20] J.-A. Kim, J. Kim, C.-S. Kang, T. B. Eom, and J. Ahn. A digital signal processing module for realtime compensation of nonlinearity in a homodyne interferometer using a field-programmable gate array. Meas. Sci. Technol., 20:017003, 2009.

[21] J. H. Galeti, C. Kitano, and M. J. Connelly. Improved synthetic-heterodyne michelson interferometer vibrometer using phase and gain control feedback. Applied $O p$ tics, 54(35):10418, 2015.

[22] A. Dandridge, A. Tveten, and T. Giallorenzi. Homodyne demodulation scheme for fiber optic sensors using phase generated carrier. IEEE Journal of Quantum Electronics, 18(10):1647-1653, 1982.

[23] G.Nomarski. Microinterféromètre différentiel à ondes polarisées. J. Phys. Radium, 16:95, 1955.

[24] C. Schonenberger and S. F. Alvarado. A differential interferometer for force microscopy. Review of Scientific Instruments, 60(10):3131-3134, 1989.

[25] F. Restagno, J. Crassous, E. Charlaix, C. Cottin-Bizonne, and M. Monchanin. A new surface forces apparatus for nanorheology. Review of Scientific Instruments, 73(6):2292-2297, 2002.

[26] P. Paolino, F. Sandoval, and L. Bellon. Quadrature phase interferometer for high resolution force spectroscopy. Rev. Sci. Instrum., 84:095001, 2013.

[27] P.L.M. Heydemann. Determination and correction of quadrature fringe measurement errors in interferometers. Appl. Opt., 20:3382, 1981.

[28] Chien-Ming Wu, Ching-shen Suy, and Gwo-Sheng Peng. Correction of nonlinearity in one-frequency optical interferometry. Meas. Sci. Technol., 7:520-524, 1996.

[29] R. Köning, G. Wimmer, and V. andWitkovský. Ellipse fitting by nonlinear constraints to demodulate quadrature homodyne interferometer signals and to determine the statistical uncertainty of the interferometric phase. Meas. Sci. Technol., page 115001, 2014.

[30] R. Köning, G. Wimmer, and V. Witkovský. The statistical uncertainty of the heydemann correction: a practical limit of optical quadrature homodyne interferometry. Meas. Sci. Technol., 26:084004, 2015.

[31] S. Leroy and E. Charlaix. Hydrodynamic interactions for the measurement of thin film elasticity. J. Fluid Mech., 674:389-407, 2011.

[32] R. Villey, E. Martinot, C. Cottin-Bizonne, M. PhanerGoutorbe, L. Leger, F. Restagno, and E. Charlaix. Effect of surface elasticity on the rheology of nanometric liquids. Phys Rev Lett, 111:215701, 2013. 